\title{
Gene expression profiling analysis to investigate the role of remote ischemic postconditioning in ischemia-reperfusion injury in rats
}

Zanxin Wang ${ }^{1,2,3^{*}}$ (D), Junmin Wen ${ }^{4,5}$, Chuzhi Zhou ${ }^{4,5}$, Zhiwei Wang ${ }^{1,2}$ and Minxin Wei ${ }^{1,2,3^{*}}$

\begin{abstract}
Background: Blood flow restoration is a definitive therapy for salvaging the myocardium following ischemic injury. Nevertheless, the sudden restoration of blood flow to the ischemic myocardium can induce ischemia-reperfusion injury (IRI).

Results: Herein, we investigated the cardioprotective effect of remote ischemic postconditioning (RPostC) through our in vivo rat model of myocardial IRI. The study included three groups: the control group, the IRI group, and the $\mid \mathrm{RI}+\mathrm{RPostC}$ group. Ischemia-reperfusion treatment led to an increase in the myocardial infarction area, which was inhibited by RPostC. In contrast to that in the control group, the myocardial apoptosis level was enhanced in the IRI group, whereas RPostC treatment decreased IRI-induced cellular apoptosis. Affymetrix Rat Gene 2.0 ST chip data identified a total of 265 upregulated genes and 267 downregulated genes between the IRI and IRI + RPostC groups. A group of differentially expressed noncoding RNAs (ncRNAs), such as MTA_TC0600002772.mm, MTA_ TC1300002394.mm, U7 small nuclear RNA (Rnu7) and RGD7543256_1, were identified. Gene Ontology (GO) enrichment analysis indicated that the positive regulation of some molecular functions, such as GTPase activity, GTP binding, cyclic-nucleotide phosphodiesterase activity and cytokine activity, may contribute to the cardioprotective role of RPostC. Moreover, pathway enrichment analysis using the Kyoto Encyclopedia of Genes and Genomes (KEGG) suggested the potential implication of the TNF signaling pathway and Toll-like receptor signaling pathway. Global signal transduction network analysis, co-expression network analysis and quantitative real-time polymerase chain reaction analysis further identified several core genes, including Pdgfra, Stat1, Lifr and Stfa3.

Conclusion: Remote ischemic postconditioning treatment can decrease IRI-mediated myocardial apoptosis by regulating multiple processes and pathways, such as GTPase activity, cytokine activity, and the TNF and Toll-like receptor signaling pathways. The potential role of the above ncRNAs and core genes in IRI-induced cardiac damage merits further study as well.
\end{abstract}

Keywords: IRI, RPostC, Apoptosis, Gene, ncRNAs

\footnotetext{
*Correspondence: zanxinwang@126.com; minxinw@126.com

'Department of Cardiac Surgery, Fuwai Hospital Chinese Academy of

Medical Sciences Shenzhen, 12 Langshan Road, Nanshan District, Shenzhen

518057, Guangdong Province, People's Republic of China

Full list of author information is available at the end of the article
}

(c) The Author(s). 2019 Open Access This article is distributed under the terms of the Creative Commons Attribution 4.0 International License (http://creativecommons.org/licenses/by/4.0/), which permits unrestricted use, distribution, and reproduction in any medium, provided you give appropriate credit to the original author(s) and the source, provide a link to the Creative Commons license, and indicate if changes were made. The Creative Commons Public Domain Dedication waiver (http://creativecommons.org/publicdomain/zero/1.0/) applies to the data made available in this article, unless otherwise stated. 


\section{Background}

Ischemia-reperfusion injury (IRI) is a phenomenon in which cell metabolism and structural damage are aggravated when blood flow is restored after myocardial ischemia and reperfusion [1-3]. Reperfusion treatment can open the infarct-related blood vessels quickly and fully restore the blood perfusion of the ischemic myocardium, rescuing the dying myocardium. Nevertheless, during the recovery of coronary blood flow, myocardial IRI can induce myocardial contraction disorders, myocardial infarct size expansion and other adverse consequences [2-4]. Our prior clinical studies reported that IRI is accompanied by different degrees of inflammatory factors, which exhibit a great influence on prognosis [5]. Hence, it is important to further investigate the mechanism underlying myocardial IRI from different perspectives and how to effectively minimize this damage through human intervention to protect the heart tissue.

Thus far, accumulating data support the association between myocardial IRI and cell stress and apoptosis; nevertheless, ischemic preconditioning, ischemic postconditioning (PostC), remote ischemic postconditioning (RPostC) and other protective measures beyond timely reperfusion are reported to reduce the myocardial damage caused by ischemia reperfusion [6-12]. As a short-term reperfusion/ischemia treatment, PostC is implemented prior to continuous reperfusion after myocardial ischemia $[6-8,10]$. RPostC refers to the protective effect seen upon the transient ischemic treatment of distant organs or tissues before the restoration of blood flow to the ischemic area $[9,13-16]$. Because RPostC avoids the clamping of coronary vessels, it achieves this myocardial protective effect through a simple and noninvasive intervention for the ischemic postconditioning of distant position and thus represents an optimistic prospect for clinical application [9, 13-16]. Nevertheless, there is insufficient experimental evidence for the mechanisms involved in the protection of the myocardium by RPostC.

In the present study, we aimed to investigate the transcriptional changes involved in cardioprotection by RPostC in rats. Based on an in vivo rat model of IRI and $\mathrm{RPostC}$, a series of assays, including gene expression profiling, functional pathway analysis, global signal transduction network analysis, co-expression network analysis and quantitative polymerase chain reaction (PCR) analysis, were conducted.

\section{Methods}

\section{IRI and IRI + RPostC model of rats}

Referring to the relative literature [17, 18], we established our rat model of IRI and RPostC treatment. A total of 24 specific pathogen-free grade Wistar male rats (Tianjin Shanchuan Red Experimental Animal
Technology Co., Ltd.) were used; the rats were housed under standard conditions of temperature $\left(22^{\circ} \mathrm{C} \pm 2{ }^{\circ} \mathrm{C}\right)$ and humidity $(40 \% \sim 70 \%)$ and a $12 \mathrm{~h} / 12 \mathrm{~h}$ light/dark cycle. Rats were randomly assigned to one of three groups: the control group, the IRI group or the IRI + $\mathrm{RPostC}$ group. The rats were anesthetized by $1 \%$ sodium pentobarbital. After endotracheal intubation, a small animal ventilator was connected. We cut the skin longitudinally along the sternum, bluntly separated the muscles with hemostatic forceps, exposed the third to fifth ribs on the left side of the sternum, and opened the chest in the fourth intercostal space on the left side of the chest. Then, we cut the pericardium and fixed the thorax with a homemade hook to fully expose the heart. The needle was inserted into the left atrial appendage and placed under the conus of the pulmonary artery. The anterior descending branch was ligated with a $6 / 0$ medical suture for $30 \mathrm{~min}$ and then loosened, and the thoracic cavity was sutured layer by layer.

In the control group, we did not block the blood vessels of the anterior descending branch after threading, and the chest was closed after $60 \mathrm{~min}$ of threading. In the IRI group, the left anterior descending coronary artery (LAD) was blocked and opened 45 min later. After 15 min of stabilization, the chest was closed. In the IRI + RPostC group, after 45 min of LAD occlusion, we performed the RPostC treatment. The left femoral artery (LFA) was clamped for $30 \mathrm{~s}$ and then opened for $30 \mathrm{~s}$. This process was repeated three times, and the chest was closed after $15 \mathrm{~min}$ of stabilization. We performed these experiments under the guidelines of animal experiments at Tianjin Medical University (2014 revision). Ethical approval for all experimental procedures was granted by the Animal Ethical and Welfare Committee at Tianjin Medical University.

\section{2,3,5-triphenylte-trazolium chloride (TTC) staining assay}

After myocardial ischemia for $45 \mathrm{~min}$ and reperfusion for $24 \mathrm{~h}$, the chest of the rat was opened. After quick freezing for $15 \mathrm{~min}$, we obtained five pieces of myocardial tissue with a thickness of approximately $1 \mathrm{~mm}$ along the long axis of the vertical heart. Next, we immediately clamped them between two slides to gently flatten them and prevent shrinkage. The samples were then placed in a 1\% TTC-phosphate buffer saline (PBS) solution at $37^{\circ}$ $\mathrm{C}$ in a constant temperature water bath for $15 \mathrm{~min}$ and then in $4 \%$ formaldehyde solution for $12 \mathrm{~h}$. The size of the myocardial infarction area, which was white or pale, was measured by ImageJ $2 \mathrm{X}$ software.

\section{Terminal deoxynucleotidyl transferase (TdT)-mediated dUTP nick end labeling (TUNEL) assay}

We used a TUNEL kit (Roche, Germany) according to the manufacturer's instructions to analyze the 
cardiomyocyte apoptosis level after reperfusion. The heart tissues were washed with PBS solution ( $\mathrm{pH} 7.4$ ). Then, the atrium was removed, and the ventricular part below the coronary artery ligation point was preserved. The vertical section of the ventricle was transversely cut into $4 \sim 6$ pieces of $1 \sim 2$-mm thick myocardial slices. The percentage of TUNEL-positive cells was determined by ImageJ $2 \mathrm{X}$ software.

\section{RNA microarray detection}

We utilized TRIzol reagent (Life Technologies) and the RNeasy Mini kit (Qiagen) to extract and purify total RNA from the cardiac left ventricle (CLV) tissue of the above three groups. Then, we used the Ambion WT Expression Kit (Affymetrix) and GeneChip WT Terminal Labeling and Controls Kit (Affymetrix) to obtain cDNA and cRNA products. Next, an Affymetrix Rat Gene 2.0 ST chip was used to hybridize the fragmented cRNA product. After staining with the GeneChip Fluidics Station 450, we applied the Affymetrix ${ }^{\oplus}$ GeneChip Command Console and robust multichip analysis algorithms to scan the microarrays and analyze the data, including Gene Ontology (GO) enrichment analysis and functional pathway analysis using the Kyoto Encyclopedia of Genes and Genomes (KEGG), as reported previously $[19,20]$. Based on the GO database, we obtained the gene function annotation of the differential genes detected above and then calculated the significance level ( $P$ value) and false positive rate (FDR) of each function by Fisher's exact test and multiple comparison test. Thus, the significant function of the gene was screened out, and a $P$ value $<0.05$ was considered significant in screening. The series entry (GSE122020, http:// www.ncbi.nlm.nih. gov/geo/query/acc.cgi? acc $=$ GSE122020) in the National Center for Biotechnology Information (NCBI) is provided.

\section{Network analysis}

According to the differentially expressed gene data, we also performed a global signal transduction network analysis to show the core genes, which have a strong correlation with other genes and play an essential role in the signaling network. In addition, based on the normalized signal intensity of RNA expression, we performed co-expression network analysis to detect potential correlations among mRNAs and identify the core genes by the degree of differences. We also performed a Venn diagram analysis (http://bioinformatics. psb.ugent.be/webtools/Venn/) to identify the common genes between the above two networks under the different limitation of degree value.

\section{Quantitative real-time polymerase chain reaction assay (qPCR)}

Based on the cDNA synthesized, we performed a StepOne Real-Time PCR System (Applied Biosystems) PCR amplification using FastStart Universal SYBR Green Master Mix (Roche Diagnostics) and the relative primers. The detailed primer sequence information is listed in Additional file 1: Table S1. The standard curve was used to assess the amplification efficiency. The melting curve assay was performed to analyze the amplification specificity. PCR reaction settings: pre-incubation step $\left(95^{\circ} \mathrm{C}\right.$, $15 \mathrm{~min})$; amplification step $\left(95^{\circ} \mathrm{C}, 10 \mathrm{~s} ; 60^{\circ} \mathrm{C}, 20 \mathrm{~s} ; 72^{\circ} \mathrm{C}\right.$, $31 \mathrm{~s} ; 40$ cycles); melting curve step $\left(95^{\circ} \mathrm{C}, 15 \mathrm{~s} ; 72^{\circ} \mathrm{C}, 15 \mathrm{~s}\right.$; $95^{\circ} \mathrm{C}, 15 \mathrm{~s}$ ). The $2^{-\Delta \Delta \mathrm{CT}}$ method was applied to calculate the relative transcript levels of candidate genes [21]. The relative expression of Glyceraldehyde-3-Phosphate Dehydrogenase (Gapdh) was utilized to normalize the expression of tested genes, including phospholipase $C$, beta 4 (Plcb4), platelet derived growth factor receptor alpha (Pdgfra), chemokine (C-C motif) receptor 1-like 1 (Ccr1l1), signal transducer and activator of transcription 1(Stat1), Jun proto-oncogene, AP-1 transcription factor subunit (Jun), Hemoglobin Subunit Alpha 2(Hba2), similar to Glutathione S-transferase A1 (GTH1) (HA subunit 1) (GST-epsilon) (GSTA1-1) (GST class-alpha) (LOC501110), stefin A3 (Stfa3), CASP8 and FADD-like apoptosis regulator (Cflar), leukemia inhibitory factor receptor alpha (Lifr), and glutathione S-transferase, mu 5 (Gstm5).

\section{Statistical analysis}

We performed one-way analysis of variance (ANOVA) / least significant difference (LSD) test or independent sample Student's t-test using BM SPSS Statistics 20 Software. When the $P$ value was $<0.05$, the differences were considered significant.

\section{Results}

\section{Effect of RPostC treatment on myocardial infarct size in} an IRI rat model

To gain insight into the potential molecular mechanism of remote ischemic postconditioning (RPostC) in ischemia/reperfusion injury (IRI), we first established an in vivo rat model of myocardial ischemia reperfusion. As shown in Fig. 1a, we did not block the blood vessels in the anterior descending branch of some rats, which were included in the study as controls. We performed RPostC treatment by three cycles of $30 \mathrm{~s}$ left femoral artery (LFA) occlusion/30 s reperfusion in rats that underwent IRI, which was induced by $45 \mathrm{~min}$ of left anterior descending coronary artery (LAD) occlusion.

Next, we carried out a histological TTC staining assay to preliminarily evaluate the impact of the ischemia/reperfusion process or RPostC treatment in rats suffering from myocardial ischemia-reperfusion injury. TTC staining was utilized to measure the myocardial infarct size. As shown in Fig. 1 b-c, we observed an increased myocardial infarction size in the IRI group compared to the control group $(P<$ $0.01)$. Nevertheless, an increased myocardial infarction area 

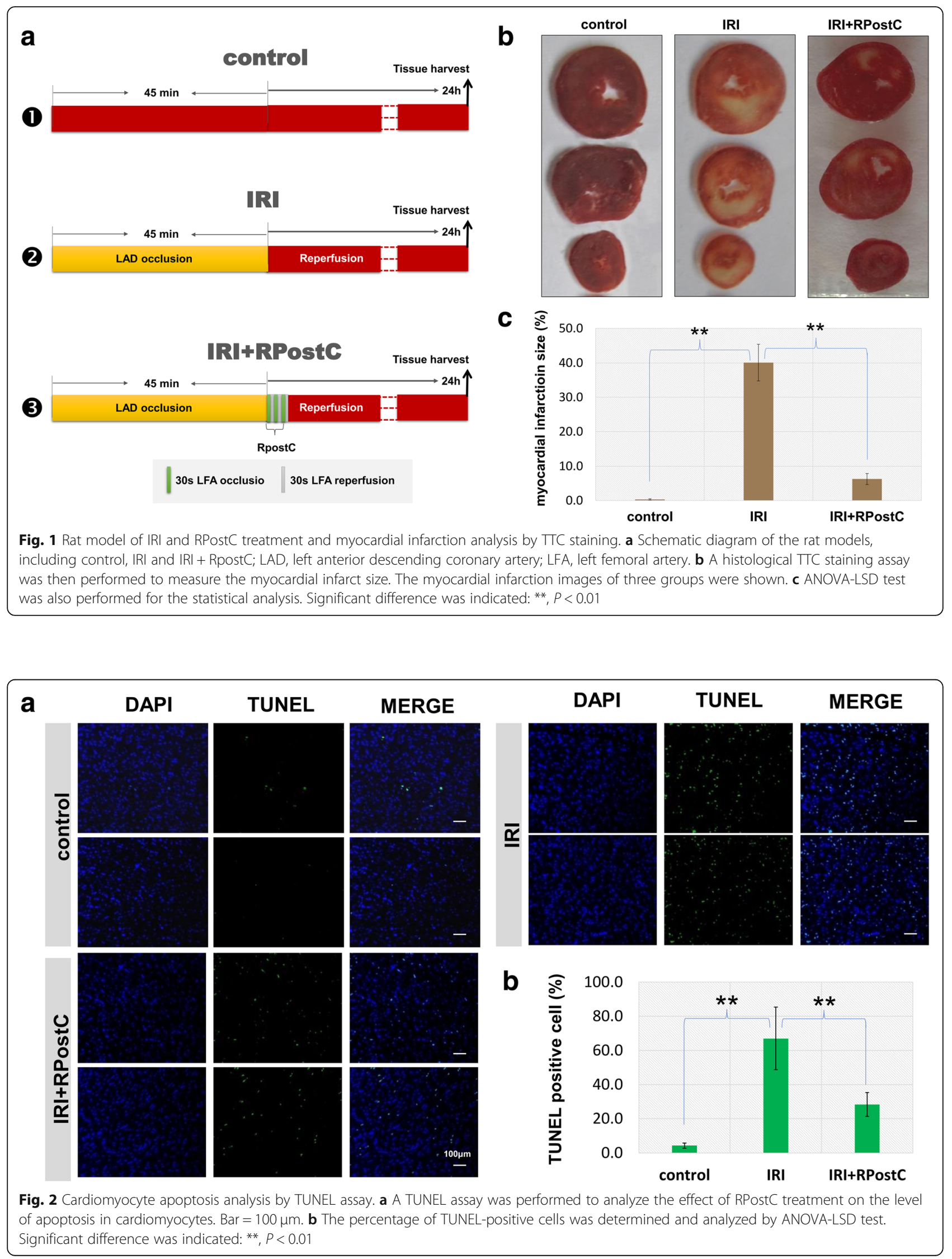
was attenuated when IRI rats were subjected to RPostC treatment in the IRI + RPostC group (Fig. 1 b-c, $P<0.01$ ). This finding indicated that RPostC treatment exhibited myocardial protection against ischemia/reperfusion injury in rats.

\section{Impact of RPostC treatment on the apoptotic level of cardiomyocytes in an IRI rat model}

We conducted the TUNEL assay to study the effect of RPostC treatment on the level of apoptosis in cardiomyocytes after reperfusion in an IRI rat model. As shown in Fig. 2 a-b, we observed an enhanced apoptotic signal in the IRI group compared with the control group $(P<0.01)$. However, a decreased apoptotic level in the IRI + RPostC group was detected when compared with that in the IRI group (Fig. 2 a-b, $P<$ 0.01). Therefore, RPostC treatment in IRI rats is capable of attenuating the high myocardial apoptosis level induced by IRI.

\section{Gene expression profiling analysis}

To investigate the molecular mechanism underlying the biological role of RPostC treatment, we conducted an Affymetrix Rat Gene 2.0 ST RNA microarray analysis using the above three rat models. Quality controls of microarray analysis are presented in Additional file 2: Figure S1. Compared to expression in the IRI group, 265 upregulated genes (with 118 ncRNA, 77 mRNA, 70 others) and 267 downregulated genes (with 182 ncRNA, 39 mRNA, 46 others) were detected in the IRI + RPostC group (Fig. 3a). To further comprehensively and intuitively present the difference between the two groups, the differentially expressed genes were subjected to hierarchical clustering and shown as a heatmap. The correlation between the samples was calculated based on the expression of the selected differential genes. Hierarchical cluster analysis data of noncoding RNAs (ncRNAs), mRNA and others are shown in Fig. 3 b-d. The red signal indicates the upregulated genes, and the green signal indicates the downregulated genes.

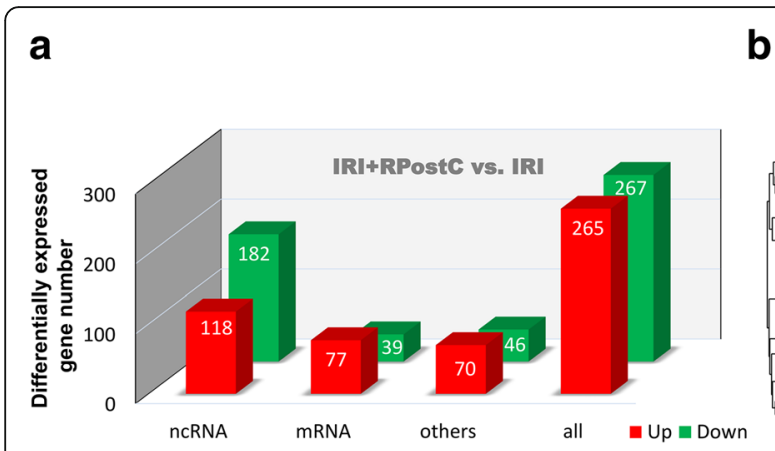

d

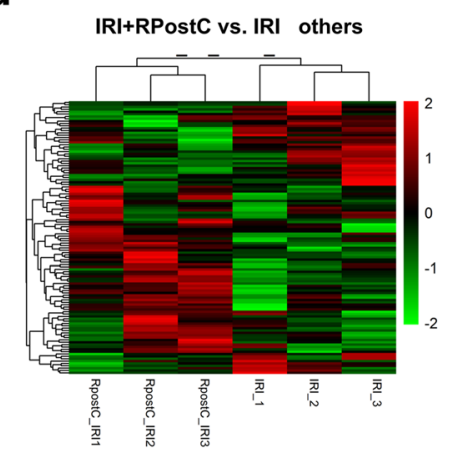

b

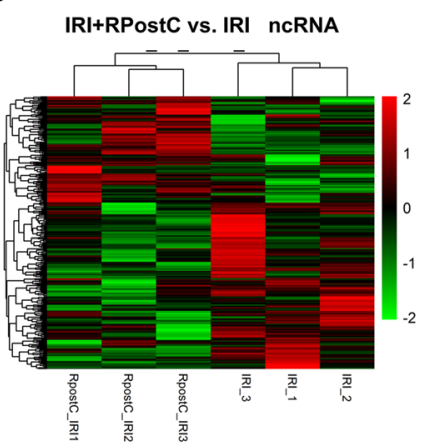

C

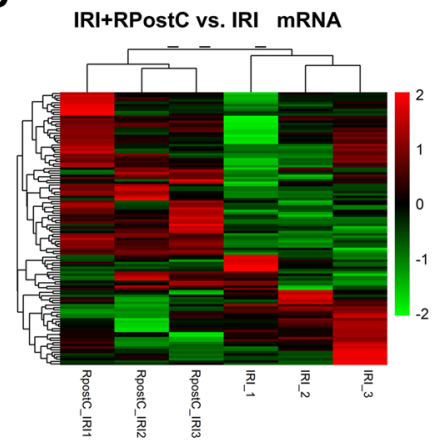

e

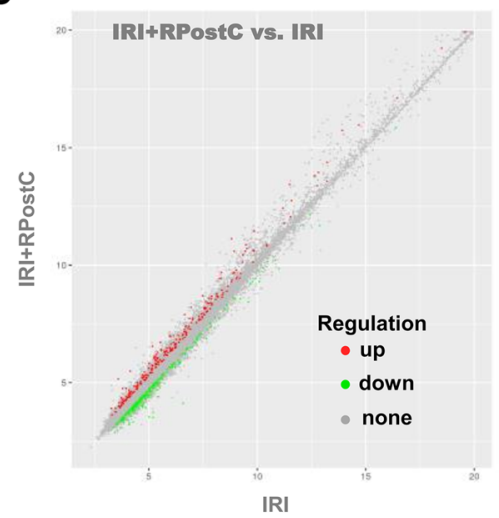

f

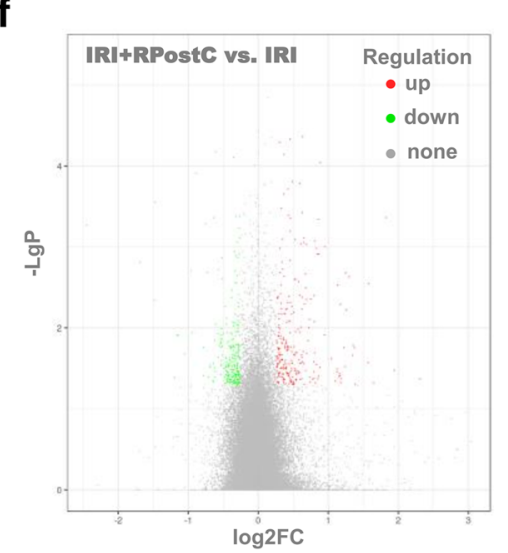

Fig. 3 Genetic difference analysis data. a An Affymetrix Rat Gene 2.0 ST RNA microarray analysis was performed. Compared to expression in the IRI group, 265 upregulated genes and 267 downregulated genes were detected in the IRI + RPostC group. b-d The differentially expressed ncRNA, mRNA and others were subjected to hierarchical clustering and shown as a heatmap, respectively. The red signal indicates the upregulated genes, and the green signal indicates the downregulated genes. e The horizontal and vertical coordinates of scatter plot represent the log 2 value of the expression levels of the two groups, respectively, showing the up-and-down distribution of the genes. $\mathbf{f} A$ volcano plot was also created based on the P-and fold-change (FC) values obtained by t-test analysis. The horizontal axis indicates the fold change of the probe, while the vertical axis represents the degree of difference in the probe $(-\log 10$ P-value, $-\mathrm{LgP})$ 
Additionally, Fig. 3e displays the scatter plot data. The horizontal and vertical coordinates represent the $\log 2$ value of the expression levels of the two groups, respectively, showing the up-and-down distribution of the genes. Furthermore, to show the significant differences between the two sets, a volcano plot (Fig. 3f) was created based on the $P$ - and fold-change (FC) values obtained by t-test analysis. The horizontal axis indicates the fold change of the probe, while the vertical axis represents the degree of difference in the probe $(-\log 10 P$-value). We listed the top ten differentially upregulated ncRNA genes, which included MTA_TC0600002772.mm, MTA_TC1300002394.mm and MTA_TC1000001004.mm, in Table 1 and the downregulated ncRNAs, which included Rnu7, MTA_ TC0500003037.mm and RGD7543256_1, in Table 2. More detailed information is shown in Additional file 3: Table S2. We also show a series of data from genetic difference analysis (Additional file 4: Fig. S2; Additional file 5: Table S3; Additional file 6: Table S4; Additional file 7: Table S5), hierarchical cluster analysis (Additional file 8: Figure S3), and scatter and volcano plots (Additional file 9: Figure S4) comparing IRI vs. control, IRI + RPostC vs. control and IRI + RPostC vs. IRI vs. control.

\section{GO enrichment and functional pathway analysis}

Next, we performed GO enrichment analysis to determine the significant, accurate, targeted gene functions of the target genes. As shown in Fig. 4a, Additional file 10: Figure S5a, and Additional file 11: Figure S6a, we found a group of upregulated genes associated with molecular functions such as GTPase activity, GTP binding, cyclic nucleotide phosphodiesterase activity, cytokine activity, the cellular response to interferon-beta, and symbiont-containing vacuole member, which is likely to be involved in the myocardial protection by $\mathrm{RPostC}$ during ischemia/reperfusion injury. Moreover, downregulated genes associated with molecular functions, such as chemokine activity, CCR chemokine receptor binding, the positive regulation of cell-cell adhesion mediated by integrin, and the formation of stress fibers, were also detected (Fig. 4b, Additional file 10: Figure S5b, and Additional file 11: Figure S6b).

Based on the bioinformatics resource KEGG, we also carried out pathway enrichment analysis. As shown in Fig. 5, when comparing the IRI group with the IRI+ RPostC group, the TNF signaling pathway and Toll-like receptor signaling pathway were identified, indicating that these pathways may be involved in the cardioprotective role of RPostC.

\section{Network analysis}

To further identify the potential core genes, which are essential in signaling networks and RNA-RNA interactions, we performed a global signal transduction network analysis and co-expression network analysis. The data from the global signal transduction network analysis (Fig. 6 and Additional file 12: Table S6) identified a total of 202 core genes, including Plcb4, Pdgfra, Ccr1 11, Stat1 and Jun. In addition, the data from the co-expression network analysis (Additional file 13: Figure S7 and Additional file 14: Table S7) identified 436 core genes, including Hba2, LOC501110, Spag9, Stfa3 and Cflar. Moreover, we performed a Venn diagram analysis to obtain a total of 64 common genes (Additional file 15: Figure S8a). We further limited the network degree value to five, and three genes, including LOC501110, Lifr and Gstm5, were identified (Additional file 15: Figure $\mathrm{S} 8 \mathrm{~b})$.

\section{Quantitative real-time PCR analysis}

We also performed quantitative real-time PCR analysis to further verify the differentially expressed mRNAs. As shown in Fig. 7, the upregulation of genes including Pdgfra, Stat1 and Lifr was detected, while the downregulation of the Stfa3 gene was observed. Further experiments targeting these genes are required.

Table 1 The top ten differentially upregulated noncoding RNAs

\begin{tabular}{|c|c|c|c|c|c|}
\hline Probe_set & FC & P.Value & Gene.Symbol & Description & Chromosome \\
\hline TC1400001307.rn.1 & 2.9650765 & 0.0268688 & MTA_TC0600002772.mm & Noncoding RNA, oocyte_clustered_small_RNA12319, complete sequence & chr14 \\
\hline TC0200002571.rn.1 & 2.5938385 & 0.0176662 & MTA_TC1300002394.mm & Noncoding transcript identified by NONCODE: Sense No Exonic & chr2 \\
\hline TC2000000948.rn.1 & 2.5457357 & 0.0218726 & MTA_TC1000001004.mm & Noncoding RNA, oocyte_clustered_small_RNA4900, complete sequence & chr20 \\
\hline TC0100003434.rn.1 & 2.2433064 & 0.0388949 & MTA_TC1900000429.mm & Noncoding transcript identified by NONCODE: Antisense & chr1 \\
\hline TC0600001997.rn.1 & 2.1809429 & 0.0322078 & MTA_TC0500000335.mm & Noncoding transcript identified by NONCODE: Sense No Exonic & chr6 \\
\hline TC1700001897.rn.1 & 1.9315169 & 0.0010048 & MTA_TC1300000124.mm & microRNA 466i & chr17 \\
\hline TC0900000059.rn.1 & 1.9266048 & 0.0122216 & RGD7738881_1 & uncharacterized LOC102554115 & chr9 \\
\hline TC1400000527.rn.1 & 1.8230086 & 0.0012326 & MTA_TC0500002485.mm & Noncoding transcript identified by NONCODE: Linc & chr14 \\
\hline TC0300001341.rn.1 & 1.749724 & 0.0204293 & RGD7622515_1 & uncharacterized LOC102554631 & chr3 \\
\hline TC1500001326.rn.1 & 1.7481686 & 0.0008483 & MTA_TC1400000136.mm & Noncoding transcript identified by NONCODE: Sense No Exonic & chr15 \\
\hline
\end{tabular}


Table 2 The top ten differentially downregulated noncoding RNAs

\begin{tabular}{llllll}
\hline Probe_set & FC & P.Value & Gene.Symbol & Description & Chromosome \\
\hline TC0900001686.rn.1 & -5.481668 & 0.0005394 & Rnu7 & U7 small nuclear RNA & chr9 \\
TC1200000831.rn.1 & -2.788872 & 0.0045801 & MTA_TC0500003037.mm & RNA for germline small RNA gsRNA59, complete sequence & chr12 \\
TC0400002518.rn.1 & -2.773747 & 0.0002789 & RGD7543256_1 & uncharacterized LOC102551678 & chr4 \\
TC0300003185.rn.1 & -2.291115 & 0.0410185 & MTA_TC0200003621.mm & predicted gene, 22,403 & chr3 \\
TC0400001897.rn.1 & -2.076163 & 0.0211123 & MTA_TC0600001556.mm & nuclear encoded rRNA 5S 166 & chr4 \\
TC0200004714.rn.1 & -1.948049 & 0.0019727 & RGD7555511_1 & uncharacterized LOC102553185 & chr2 \\
TC0300004193.rn.1 & -1.917423 & 0.0114548 & MTA_TC0200004648.mm & microRNA 3098 (Mir3098), microRNA & chr3 \\
TC0X00001589.rn.1 & -1.850113 & 0.0001236 & MTA_TC0X00001571.mm & predicted gene, 22,359 & chrX \\
TC0100006120.rn.1 & -1.723291 & 0.0389854 & MTA_TC0700003802.mm & microRNA 3965, microRNA 3965 (Mir3965), microRNA & chr1 \\
TC1300000971.rn.1 & -1.722596 & 0.0173776 & Mir664-2 & microRNA mir-664-2 & chr13 \\
\hline FC fold chang & & & & &
\end{tabular}

FC fold change

\section{Discussion}

The protective role of ischemic preconditioning and postconditioning, to varying degrees, has previously been identified in several studies using animal models. For example, it has been shown that ischemic preconditioning treatment in myocardial IRI rats exhibits a more powerful protective effect than limb remote ischemic postconditioning treatment during myocardial ischemia-reperfusion injury [22]. Compared to classic postconditioning, remote postconditioning treatment has a greater potential role in reducing the infarct size in a New Zealand white male rabbit model [23]. In the present study, we utilized an in vivo rat model of IRI to evaluate the role of RPostC treatment after myocardial ischemia-reperfusion injury. We found that RPostC treatment resulted in a reduction in the IRI-induced myocardial infarction area. The TUNEL assay data further indicated the involvement of an antiapoptotic effect in the protective mechanism of RPostC treatment after myocardial injury. These data are consistent with relevant studies in other animal models. For instance, myocardial infarct size was also significantly decreased in the RPostC group compared with the control group after acute myocardial infarction in pigs [24].

The molecular mechanism of the protective effect of RPostC treatment remains largely unclear. Multiple processes, such as the inflammatory response, oxidative stress, and leukocyte infiltration, and gasotransmitters ( $\mathrm{NO}, \mathrm{H}_{2} \mathrm{~S}$ and $\mathrm{CO}$ ) may be potentially implicated in the role of RPostC in myocardial ischemia-reperfusion injury [9, 13, 25-29]. For example, the inhibition of receptors for advanced glycation end products (RAGE) and high-mobility group box1 (HMGB1) expression and activation of the PI3K/Akt signaling pathway have been linked to the extenuated ischemic reperfusion injury in a mouse model [29]. Our current study revealed that chemokine activity, CCR chemokine receptor binding and cytokine activity may be associated with the myocardial protective role of RPostC treatment after ischemia/reperfusion injury in a rat model, which supports the idea that the inflammatory response is indeed an essential factor for reducing ischemia-reperfusion-induced cardiac damage. Moreover, our findings offer a potential functional link between GTPase activity, GTP binding issues and cardioprotection by RPostC treatment.

The Toll-like receptor signaling pathway is related to the myocardial immune response and ischemia/reperfusion [30-33]. It has been reported that the postconditioning of sevoflurane confers a neuroprotective role in a rat model of transient global cerebral ischemia, and the Toll-like receptor-4 (TLR4)/nuclear factor kappa B $(\mathrm{NF}-\mathrm{kB})$ pathway and subsequent anti-inflammation activity may be implicated in this process [34]. The TLR4/ NF- $\mathrm{kB}$ signaling pathway has also been reported to be associated with vaspin-mediated cardioprotective effects on myocardial ischemia/reperfusion injury [35]. Herein, we further utilized an Affymetrix Rat Gene 2.0 ST chip to perform gene expression profiling, GO enrichment and functional pathway analysis. We also observed the potential role of the Toll-like receptor signaling pathway in the $\mathrm{RPostC}$-mediated cardioprotective process.

Additionally, it is worth mentioning that a we are the first to identify a group of differentially expressed ncRNA genes. Our global signal transduction network analysis and co-expression network analysis also identified several core genes, such as Pdgfra, Stat1, Lifr and Stfa3. It is meaningful to study the expression of RPostC-associated ncRNAs and core genes and to investigate their functional relationship with the serum or myocardial level of inflammatory-related biomarkers, which may contribute to the optimization of the details of the RPostC protocol and an increase in survival duration. Considering the role of coronary circulation in the cardioprotection process [36], more relative molecular investigations are needed to further study the functional role of the expression of the targeting genes in the cardiomyocytes and other protected cardiac cells. 

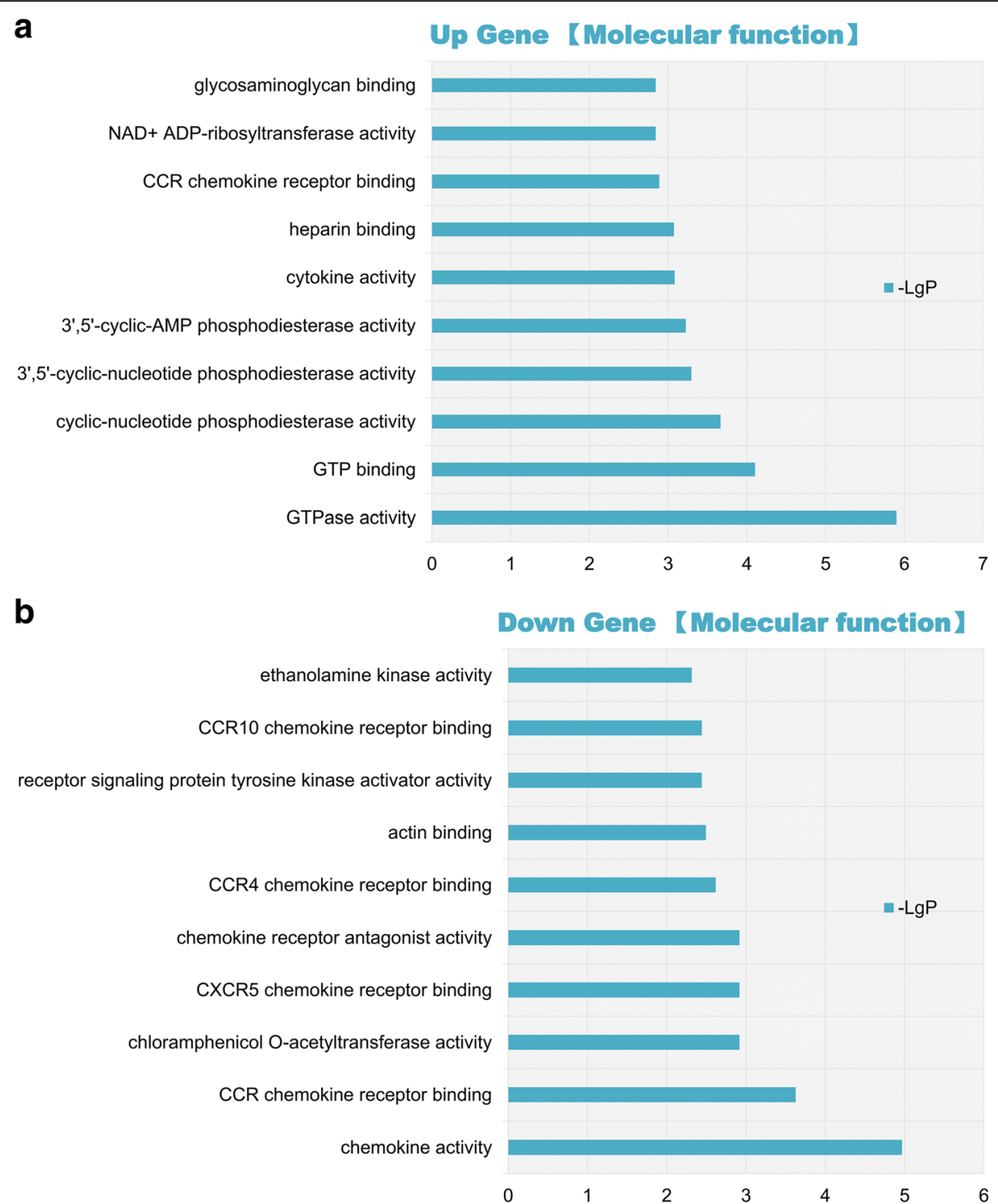

Fig. $4 \mathrm{GO}$ Enrichment analysis of molecular function. To determine the significant, accurate, targeted gene functions of the target genes, GO enrichment analysis was performed. A group of upregulated genes (a) and downregulated genes $(\mathbf{b})$ associated with molecular functions were shown. The vertical axis indicates the pathway names, while the horizontal axis represents the degree of difference in the probe $(-\log 10 P$-value, $-\operatorname{LgP})$

Ischemic postconditioning treatment exhibits a protective role on the heart as well as some other ischemia-sensitive organs via a complex mechanism. For instance, RPostC treatment has an influence on cerebral ischemia-reperfusion injury [37-39]. The T-LAK-cell -originated protein kinase (TOPK)/phosphatase and tensin homolog deleted on chromosome ten (PTEN)/Akt signaling pathway is associated with the protective effects of RPostC treatment after renal ischemia/reperfusion injury [40]. RPostC treatment exhibits a protective effect on limb ischemia-reperfusion-induced gastric mucosal injury, in which it is implicated in anti-inflammatory and antioxidant activity [41]. Ischemic postconditioning treatment also reportedly has a protective effect on renal ischemia and reperfusion injury in rats via the modulation of the anti-inflammatory response [42] and on the ischemia-reperfusion injury of rat liver graft [43]. The hypoxia inducible factor 1 alpha (HIF-1 $\alpha$ )/microRNA-21 (miR-21) axis may contribute to the protective role of the ischemic postconditioning approach [44]. Although remote ischemic postconditioning treatment is a feasible operation strategy, it is difficult to control the degree and timing of ischemic postconditioning intervention. The myocardial protection process triggered by RPostC treatment is closely associated with the length of ischemic and intervention time and the physiological features of different ischemic tissues and organs. 


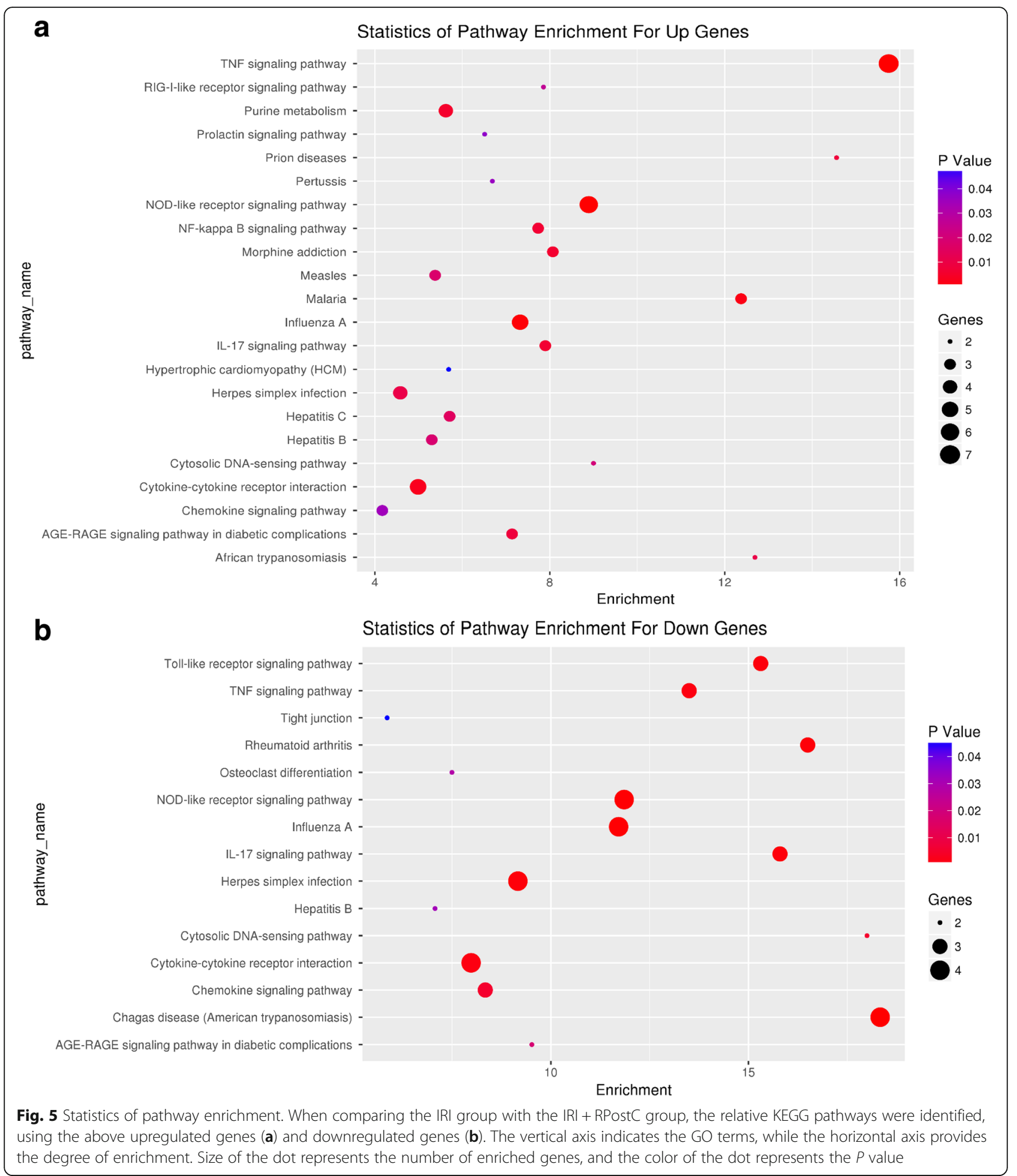

The combination of natural pharmaceuticals, such as troxerutin, and RPostC treatment has also been considered [27]. Support for pharmacological and/or ischemic postconditioning requires additional clinical trial data [45]. Postconditioning of endomorphin-1 in a rat model also reportedly decreases myocardial cell apoptosis and tissue injury, possibly through the regulation of inflammatory or oxidative stress [46]. All of these methods will be useful to reduce IRI-mediated postoperative complications. Given the complicated mechanism of myocardial damage after ischemia and reperfusion [47, 48], additional molecular evidence regarding the role of 


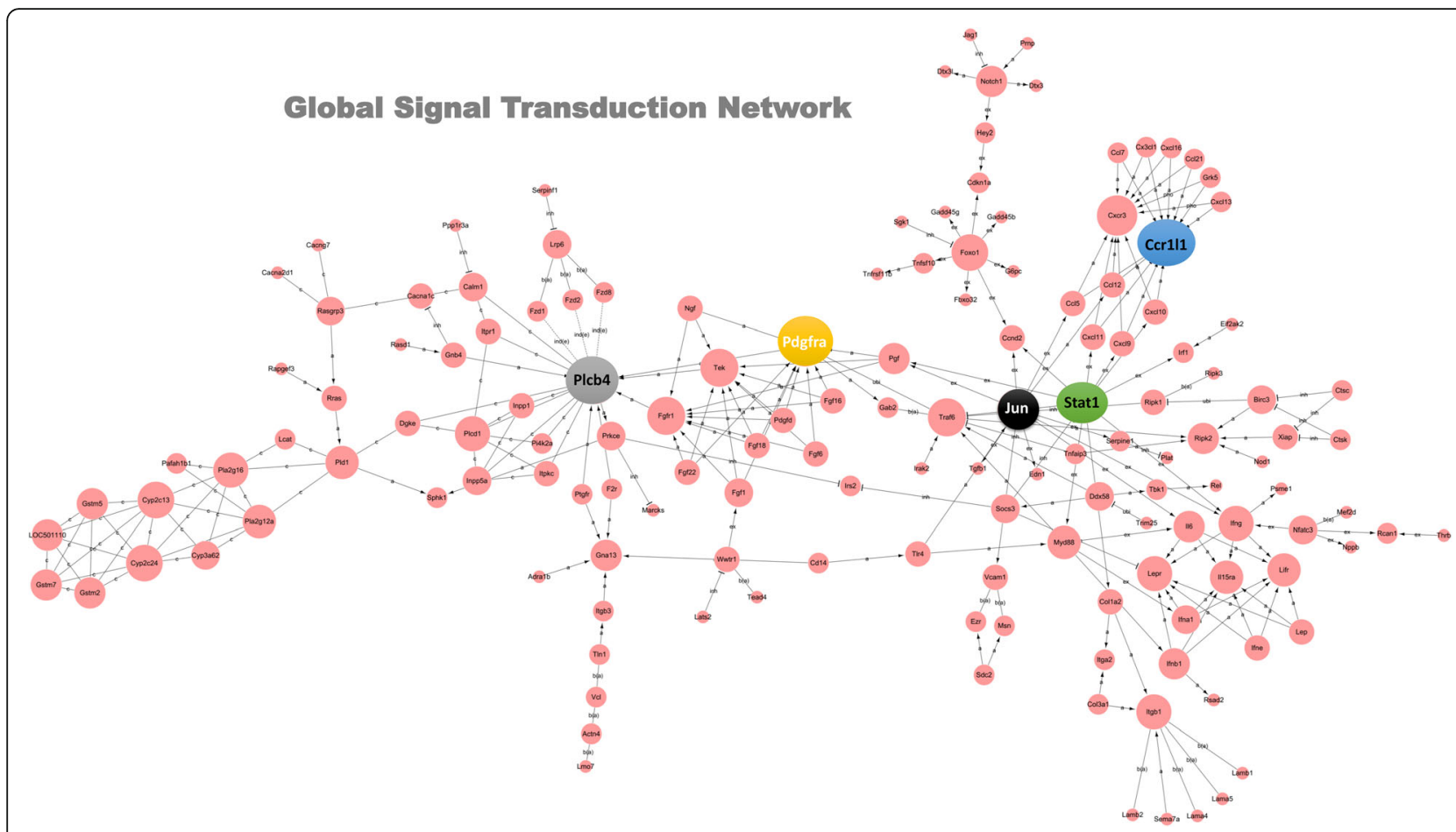

Fig. 6 Global signal transduction network data. Based on the differentially expressed gene data, we performed a global signal transduction network analysis to identify a total of 202 core genes, including PIcb4, Pdgfra, Ccr1 11, Stat1 and Jun.

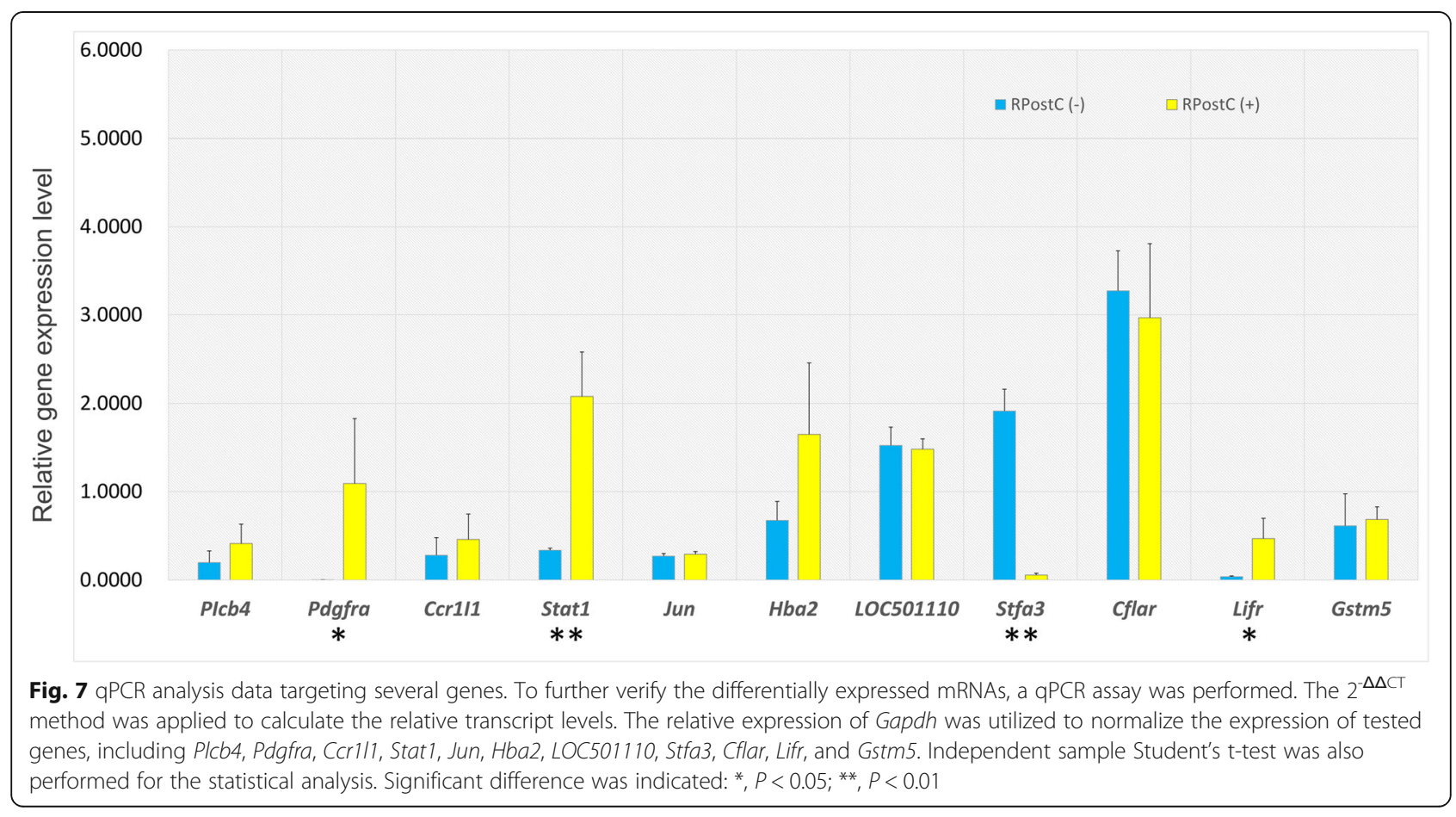


RPostC in IRI-induced myocardial necrosis or pyroptosis is needed. In addition, considering the critical issues for the translation from experimental cardioprotection studies to clinical patient benefit [49], more results of the clinical trials or molecular tests from the patients are required to support our gene expression profiling analysis data from the rat models.

\section{Conclusions}

To ensure optimal myocardial protection and minimize other influencing factors, it is meaningful to investigate the specific molecular mechanisms underlying remote ischemic postconditioning, optimize specific clinical implementation methods, discover relevant biomarkers, develop related drugs, and target the drugs to their corresponding signal transduction pathways. The potential protective effect of RPostC and its association with various ncRNAs, GTPase activity, cytokine activity, the TNF and Toll-like receptor signaling pathways, and core genes such as Pdgfra, Stat1, Lifr and Stfa3 merit further molecular analysis.

\section{Additional files}

Additional file 1: Table S1. The primer sequences used in the $\mathrm{qPCR}$ assay. (XLSX $31 \mathrm{~kb}$ )

Additional file 2: Figure S1. Quality controls of the microarray analysis. a Chip-box data; b chip-histogram data; c hybrid quality control; d negative-positive quality control. (TIF $7165 \mathrm{~kb}$ )

Additional file 3: Table S2. The differently expressed genes identified by IRI + RPostC vs. IRI vs. control comparison. (XLSX 203 kb)

Additional file 4: Figure S2. An Affymetrix Rat Gene 2.0 ST RNA microarray analysis was performed for the genetic difference analysis data. A number of upregulated genes and downregulated genes were detected in the comparisons of IRI vs. control (a); IRI + RPostC vs. control (b); IRI + RPostC vs. IRI vs. control (c (TIF 8798 kb)

Additional file 5: Table S3. The differently expressed genes identified by IRI vs. control comparison. (XLSX $2576 \mathrm{~kb}$ )

Additional file 6: Table S4. The differently expressed genes identified by IRI + RPostC vs. control comparison. (XLSX 3221 kb)

Additional file 7: Table S5. The differently expressed genes identified by IRI + RPostC vs. control comparison. (XLSX 3603 kb)

Additional file 8: Figure S3. The differentially expressed ncRNA, mRNA and others in the comparisons of IRI vs. control (a-c), IRI + RPostC vs. control (d-e), IRI + RPostC vs. IRI vs. control (f-h) were subjected to hierarchical clustering and shown as a heatmap, respectively. The red signal indicates the upregulated genes, and the green signal indicates the downregulated genes. (TIF $17130 \mathrm{~kb}$ )

Additional file 9: Figure S4. The horizontal and vertical coordinates of scatter plots in the comparisons of IRI vs. control (a), IRI + RPostC vs. control (b) represent the log2 value of the expression levels of the two groups, respectively, showing the up-and-down distribution of the genes. The volcano plots in the comparisons of $\mid \mathrm{RI}$ vs. control (c), IRI + RPostC vs. control (d) was also created based on the $P$ - and fold-change (FC) values obtained by t-test analysis. The horizontal axis indicates the fold change of the probe, while the vertical axis represents the degree of difference in the probe $(-\log 10$ P-value, - LgP). (TIF $33038 \mathrm{~kb}$ )

Additional file 10: Figure S5. GO Enrichment analysis of biological processes. A group of upregulated genes (a) and downregulated genes (b) associated with biological processes were shown. The vertical axis indicates the pathway names, while the horizontal axis represents the degree of difference in the probe (-log10 P-value, -LgP). (TIF 48577 kb)

Additional file 11: Figure S6. GO Enrichment analysis of cellular components. A group of upregulated genes (a) and downregulated genes (b) associated with cellular components were shown. The vertical axis indicates the pathway names, while the horizontal axis represents the degree of difference in the probe (-log10 P-value, -LgP). (TIF $52073 \mathrm{~kb}$ )

Additional file 12: Table S6. Gene information from the global signal transduction network analysis. (XLSX $37 \mathrm{~kb}$ )

Additional file 13: Figure S7. Based on the normalized signal intensity of RNA expression, co-expression network analysis was performed to detect potential correlations among mRNAs and identify the core genes by the degree of differences. (TIF $31248 \mathrm{~kb}$ )

Additional file 14: Table S7. Gene information from the co-expression network analysis. (XLSX $67 \mathrm{~kb}$ )

Additional file 15: Figure S8. The Venn diagram analysis data. a All core genes identified by global signal transduction network analysis and co-expression network analysis. b All core genes identified by the two network analyses with a degree number $>=5$. (TIF $12766 \mathrm{~kb}$ )

\section{Abbreviations}

IRI: Ischemia reperfusion injuryRPostCRemote ischemic postconditioningncRNAsnon-coding RNAsRnu7U7 small nuclear RNAPostClschemic postconditioningLADLeft anterior descending coronary arteryLFALeft femoral artery TTC2,3,5-triphenylte-trazolium chloridePBSPhosphate buffer salineTUNELTerminal dexynucleotidyl transferase (TdT)-mediated dUTP nick end labelingCLVCardiac left ventricleGOGene OntologyKEGGKyoto Encyclopedia of Genes and GenomesNCBINational center for biotechnology informationqPCRquantitative real-time polymerase chain reaction assayGapdhGlyceraldehyde-3-Phosphate DehydrogenasePIcb4Phospholipase C, beta 4PdgfraPlatelet derived growth factor receptor alphaCcr1IChemokine (C-C motif) receptor 1-like 1Stat1Signal transducer and activator of transcription 1JunJun proto-oncogene,AP-1 transcription factor subunitHba2Hemoglobin Subunit Alpha 2LOC501110Similar to Glutathione S-transferase A1 (GTH1) (HA subunit 1) (GST-epsilon) (GSTA11) (GST class-alpha)Stfa3Stefin A3CflarCASP8 and FADD-like apoptosis regulatorLifrLeukemia inhibitory factor receptor alphaGstm5Glutathione Stransferase, mu 5ANOVAOne-way analysis of varianceLSDLeast Significant DifferenceFDRFalse positive rateFCFold changeRAGEInhibited receptor for advanced glycation end productsHMGB1High-mobility group box1TLR4TollLike Receptor-4NF-KBNuclear Factor Kappa BTOPKT-LAK-cell-originated protein kinasePTENPhosphatase and tensin homolog deleted on chromosome tenHIF-1aHypoxia inducible factor 1 alphamiR-21microRNA-21

\section{Acknowledgements}

The authors appreciate Rong Guo of Beijing Cnkingbio Biotechnology Co.LTD for the bioinformatics assistance. The authors also thank American Journal Experts (https://www.aje.com/) for help with English usage.

\section{Funding}

This study was funded by the National Natural Science Foundation of China (No. 81600208 (Wang Z) and 81570256 (Wei M)) (IRI model, RNA microarray and QPCR assay); the Tianjin Municipal Science and Technology Commission [No. 14ZCZDSY00023], Medical Scientific Research Foundation of Guangdong Province of China (A2018019) , Science and Technology project of Shenzhen of China [grant number JCYJ20180302173909492 (Wang Z) and JCYJ20180508152222104 (Wei M)] and the "Sanming" Project of Medicine in Shenzhen, P. R. China (TTC staining, TUNEL and network assays).

\section{Availability of data and materials}

All data and materials are available on request.

\section{Authors' contributions}

ZaW and MW contribution to conception and design. ZaW and JW prepared the rat model of IRI for RNA microarray detection. ZaW, CZ and ZhW performed the TTC staining, TUNEL and q-PCR assays. ZaW analyzed the data and wrote the manuscript. MW supervised the experiments, reviewed and revised the manuscript critically for important intellectual content. All authors approved the final manuscript and agreed to be accountable for all aspects 
of the work in ensuring that questions related to the accuracy or integrity of any part of the work are appropriately investigated and resolved.

\section{Ethics approval and consent to participate}

All experimental procedures and ethics approval were approved by the Animal Ethical and Welfare Committee (AEWC) in Tianjin Medical University. Under the guidelines of animal experiments in Tianjin Medical University (2014 revision), we conducted our animal experiments. Every effort was made to minimize animal suffering. "No applicable" for the consent to participate.

\section{Competing interests}

The authors declare that they have no competing interests.

\section{Publisher's Note}

Springer Nature remains neutral with regard to jurisdictional claims in published maps and institutional affiliations.

\section{Author details}

${ }^{1}$ Department of Cardiac Surgery, Fuwai Hospital Chinese Academy of Medical Sciences Shenzhen, 12 Langshan Road, Nanshan District, Shenzhen 518057, Guangdong Province, People's Republic of China. ${ }^{2}$ Department of Cardiac Surgery, Shenzhen Sun Yat-sen Cardiovascular Hospital, Shenzhen, People's Republic of China. ${ }^{3}$ Department of Cardiovascular Surgery, Tianjin Medical University General Hospital, Tianjin, People's Republic of China. ${ }^{4}$ Department of Intensive Care, Fuwai Hospital Chinese Academy of Medical Sciences Shenzhen, Shenzhen, Guangdong, People's Republic of China. ${ }^{5}$ Department of Intensive Care, Shenzhen Sun Yat-sen Cardiovascular Hospital, Shenzhen, People's Republic of China.

\section{Received: 11 October 2018 Accepted: 29 April 2019}

\section{Published online: 09 May 2019}

\section{References}

1. Hausenloy DJ, Yellon DM. Ischaemic conditioning and reperfusion injury. Nat Rev Cardiol. 2016;13(4):193-209.

2. Hausenloy DJ, Botker HE, Engstrom T, Erlinge D, Heusch G, Ibanez B, Kloner RA, Ovize M, Yellon DM, Garcia-Dorado D. Targeting reperfusion injury in patients with ST-segment elevation myocardial infarction: trials and tribulations. Eur Heart J. 2017;38(13):935-41.

3. Heusch G, Gersh BJ. The pathophysiology of acute myocardial infarction and strategies of protection beyond reperfusion: a continual challenge. Eur Heart J. 2017;38(11):774-84

4. Bulluck $H$, Yellon DM, Hausenloy DJ. Reducing myocardial infarct size: challenges and future opportunities. Heart. 2016;102(5):341-8.

5. Wang Z, Shao J, Zhou Q, Liu J, Zhu Y, Yang J, Wei M. The -251A>T polymorphism of interleukin-8 is associated with longer mechanical ventilation and hospital staying after coronary surgery. Cytokine. 2010;50(3): 268-72

6. Heusch G, Rassaf T. Time to give up on Cardioprotection? A critical appraisal of clinical studies on ischemic pre-, post-, and remote conditioning. Circ Res. 2016;119(5):676-95.

7. Xia JG, Xu FF, Qu Y, Song DG, Shen H, Liu XH. Atorvastatin postconditioning attenuates myocardial ischemia reperfusion injury via inhibiting endoplasmic reticulum stress-related apoptosis. Shock. 2014:42(4): 365-71

8. Xie L, Pi X, Wang Z, He J, Willis MS, Patterson C. Depletion of PHD3 protects heart from ischemia/reperfusion injury by inhibiting cardiomyocyte apoptosis. J Mol Cell Cardiol. 2015:80:156-65.

9. Xu J, Sun S, Lu X, Hu X, Yang M, Tang W. Remote ischemic pre- and postconditioning improve postresuscitation myocardial and cerebral function in a rat model of cardiac arrest and resuscitation. Crit Care Med. 2015;43(1):e12-8.

10. Heusch $\mathrm{G}$. Treatment of myocardial ischemia/reperfusion injury by ischemic and pharmacological Postconditioning. Compr Physiol. 2015;5(3):1123-45.

11. Hausenloy DJ, Barrabes JA, Botker HE, Davidson SM, Di Lisa F, Downey J, Engstrom T, Ferdinandy P, Carbrera-Fuentes HA, Heusch G, et al. Ischaemic conditioning and targeting reperfusion injury: a 30 year voyage of discovery. Basic Res Cardiol. 2016;111(6):70.

12. Heusch G. 25 years of remote ischemic conditioning: from laboratory curiosity to clinical outcome. Basic Res Cardiol. 2018;113(3):15.
13. Andreadou I, lliodromitis EK, Rassaf T, Schulz R, Papapetropoulos A, Ferdinandy P. The role of gasotransmitters $\mathrm{NO}, \mathrm{H} 2 \mathrm{~S}$ and $\mathrm{CO}$ in myocardial ischaemia/reperfusion injury and cardioprotection by preconditioning, postconditioning and remote conditioning. Br J Pharmacol. 2015;172(6): 1587-606.

14. Kerendi F, Kin H, Halkos ME, Jiang R, Zatta AJ, Zhao ZQ, Guyton RA, VintenJohansen J. Remote postconditioning. Brief renal ischemia and reperfusion applied before coronary artery reperfusion reduces myocardial infarct size via endogenous activation of adenosine receptors. Basic Res Cardiol. 2005; 100(5):404-12.

15. Heusch G, Botker HE, Przyklenk K, Redington A, Yellon D. Remote ischemic conditioning. J Am Coll Cardiol. 2015;65(2):177-95.

16. Kleinbongard P, Skyschally A, Heusch G. Cardioprotection by remote ischemic conditioning and its signal transduction. Pflugers Arch. 2017:469(2) 159-81.

17. Botker HE, Hausenloy D, Andreadou I, Antonucci S, Boengler K, Davidson SM, Deshwal S, Devaux Y, Di Lisa F, Di Sante M et al. Practical guidelines for rigor and reproducibility in preclinical and clinical studies on cardioprotection 2018;113(5):39.

18. Baars T, Skyschally A, Klein-Hitpass L, Cario E, Erbel R, Heusch G, Kleinbongard P. microRNA expression and its potential role in cardioprotection by ischemic postconditioning in pigs. Pflugers Arch. 2014; 466(10):1953-61.

19. Wang R, Su C, Wang X, Fu Q, Gao X, Zhang C, Yang J, Yang X, Wei M. Global gene expression analysis combined with a genomics approach for the identification of signal transduction networks involved in postnatal mouse myocardial proliferation and development. Int J Mol Med. 2018;41(1): $311-21$.

20. Cui X, Zhao C, Yao X, Qian B, Su C, Ren Y, Yao Z, Gao X, Yang J. SND1 acts as an anti-apoptotic factor via regulating the expression of IncRNA UCA1 in hepatocellular carcinoma. RNA Biol. 2018;15(10):1364-75.

21. Livak KJ, Schmittgen TD. Analysis of relative gene expression data using real-time quantitative PCR and the 2(-Delta Delta C(T)) method. Methods. 2001;25(4):402-8.

22. Zhang JQ, Wang Q, Xue FS, Li RP, Cheng Y, Cui XL, Liao X, Meng FM. Ischemic preconditioning produces more powerful anti-inflammatory and cardioprotective effects than limb remote ischemic postconditioning in rats with myocardial ischemia-reperfusion injury. Chin Med J. 2013;126(20):3949-55.

23. Gritsopoulos G, Iliodromitis EK, Zoga A, Farmakis D, Demerouti E, Papalois A, Paraskevaidis IA, Kremastinos DT. Remote postconditioning is more potent than classic postconditioning in reducing the infarct size in anesthetized rabbits. Cardiovasc Drugs Ther. 2009;23(3):193-8.

24. Andreka G, Vertesaljai M, Szantho G, Font G, Piroth Z, Fontos G, Juhasz ED Szekely L, Szelid Z, Turner MS, et al. Remote ischaemic postconditioning protects the heart during acute myocardial infarction in pigs. Heart. 2007; 93(6):749-52.

25. Steffens S, Montecucco F, Mach F. The inflammatory response as a target to reduce myocardial ischaemia and reperfusion injury. Thromb Haemost. 2009;102(2):240-7

26. Kim YH, Yoon DW, Kim JH, Lee JH, Lim CH. Effect of remote ischemic postconditioning on systemic inflammatory response and survival rate in lipopolysaccharide-induced systemic inflammation model. J Inflamm (Lond). 2014;11:16.

27. Badalzadeh R, Baradaran B, Alihemmati A, Yousefi B, Abbaszadeh A. Troxerutin preconditioning and ischemic Postconditioning modulate inflammatory response after myocardial ischemia/reperfusion injury in rat model. Inflammation. 2017:40(1):136-43.

28. Cohen MV, Downey JM. Signalling pathways and mechanisms of protection in pre- and postconditioning: historical perspective and lessons for the future. Br J Pharmacol. 2015;172(8):1913-32.

29. Wang X, Wang J, Tu T, lyan Z, Mungun D, Yang Z. Remote ischemic Postconditioning protects against myocardial ischemia-reperfusion injury by inhibition of the RAGE-HMGB1 pathway. 2018;2018:4565630.

30. Li M, Liu J, Bi Y, Chen J, Zhao L. Potential medications or compounds acting on toll-like receptors in cerebral ischemia. Curr Neuropharmacol. 2018;16(2): 160-75.

31. Vilahur $G$, Badimon L. Ischemia/reperfusion activates myocardial innate immune response: the key role of the toll-like receptor. Front Physiol. 2014;5:496.

32. Ha T, Liu L, Kelley J, Kao R, Williams D, Li C. Toll-like receptors: new players in myocardial ischemia/reperfusion injury. Antioxid Redox Signal. 2011;15(7): 1875-93. 
33. Wang Y, Abarbanell AM, Herrmann JL, Weil BR, Poynter J, Manukyan MC, Crisostomo PR, Meldrum DR. Toll-like receptor signaling pathways and the evidence linking toll-like receptor signaling to cardiac ischemia/reperfusion injury. Shock. 2010;34(6):548-57.

34. Hwang JW, Jeon YT, Lim YJ, Park HP. Sevoflurane Postconditioning-induced anti-inflammation via inhibition of the toll-like Receptor-4/nuclear factor kappa B pathway contributes to neuroprotection against transient global cerebral ischemia in rats, vol. 18; 2017. p. 11.

35. Yuan L, Dai X, Fu H, Sui D, Lin L, Yang L, Zha P, Wang X, Gong G. Vaspin protects rats against myocardial ischemia/reperfusion injury (MIRI) through the TLR4/NF-kappaB signaling pathway. Eur J Pharmacol. 2018;835:132-9.

36. Heusch G. The coronary circulation as a target of Cardioprotection. Circ Res. 2016;118(10):1643-58.

37. Liu Q, Zhou S, Wang Y, Qi F, Song Y, Long S. A feasible strategy for focal cerebral ischemia-reperfusion injury: remote ischemic postconditioning. Neural Regen Res. 2014;9(15):1460-3.

38. Xie R, Li J, Zhao H. The underlying mechanisms involved in the protective effects of ischemic postconditioning. Cond Med. 2018;1(2):73-9.

39. Ren C, Yan Z, Wei D, Gao X, Chen X, Zhao H. Limb remote ischemic postconditioning protects against focal ischemia in rats. Brain Res. 2009; 1288:88-94.

40. Gao S, Zhu Y, Li H, Xia Z, Wu Q, Yao S, Wang T, Yuan S. Remote ischemic postconditioning protects against renal ischemia/reperfusion injury by activation of T-LAK-cell-originated protein kinase (TOPK)/PTEN/Akt signaling pathway mediated anti-oxidation and anti-inflammation. Int Immunopharmacol. 2016:38:395-401.

41. Wang T, Zhou YT, Chen XN, Zhu AX, Wu BH. Remote ischemic postconditioning protects against gastric mucosal lesions in rats. World J Gastroenterol. 2014;20(28):9519-27.

42. Chen $H$, Wang L, Xing BZ, Liu XH, Chen ZY, Weng XD, Qiu T, Liu L. Ischemic postconditioning attenuates inflammation in rats following renal ischemia and reperfusion injury. Exp Ther Med. 2015;10(2):513-8.

43. Li JH, Jia JJ, Shen W, Chen SS, Jiang L, Xie HY, Zhou L, Zheng SS. Optimized postconditioning algorithm protects liver graft after liver transplantation in rats. Hepatobiliary Pancreat Dis Int. 2018;17(1):32-8.

44. Jia Z, Lian W, Shi H, Cao C, Han S, Wang K, Li M, Zhang X. Ischemic Postconditioning protects against intestinal ischemia/reperfusion injury via the HIF-1alpha/miR-21 Axis. Sci Rep. 2017;7(1):16190.

45. Buchholz B, Donato M, D'Annunzio V, Gelpi RJ. Ischemic postconditioning: mechanisms, comorbidities, and clinical application. Mol Cell Biochem. 2014; 392(1-2): $1-12$

46. Zhang WP, Zong QF, Gao Q, Yu Y, Gu XY, Wang Y, Li ZH, Ge M. Effects of endomorphin-1 postconditioning on myocardial ischemia/reperfusion injury and myocardial cell apoptosis in a rat model. Mol Med Rep. 2016;14(4): 3992-8.

47. Jia C, Chen H, Zhang J, Zhou K, Zhuge Y, Niu C, Qiu J, Rong X, Shi Z, Xiao J, et al. Role of pyroptosis in cardiovascular diseases. Int Immunopharmacol. 2019;67:311-8

48. Wu MY, Yiang GT, Liao WT, Tsai AP, Cheng YL, Cheng PW, Li CY, Li CJ. Current mechanistic concepts in ischemia and reperfusion injury. Cell Physiol Biochem. 2018;46(4):1650-67.

49. Heusch G. Critical issues for the translation of Cardioprotection. Circ Res. 2017;120(9):1477-86

\section{Ready to submit your research? Choose BMC and benefit from:}

- fast, convenient online submission

- thorough peer review by experienced researchers in your field

- rapid publication on acceptance

- support for research data, including large and complex data types

- gold Open Access which fosters wider collaboration and increased citations

- maximum visibility for your research: over $100 \mathrm{M}$ website views per year

At $\mathrm{BMC}$, research is always in progress.

Learn more biomedcentral.com/submissions 